\title{
Differential expression of immune-related genes in the bursa of Fabricius of two inbred chicken lines following infection with very virulent infectious bursal disease virus
}

\begin{abstract}
Among different inbred chickens' lines, we previously showed that lines $\mathrm{P}$ and $\mathrm{N}$ of Institute for Animal Health, Compton, UK are the most susceptible and the least affected lines, respectively, following infection with very virulent infectious bursal disease virus (vvIBDV). In this study, the differential expressions of 29 different immune-related genes were characterized. Although, birds from both lines succumbed to infection, line $\mathrm{P}$ showed greater bursal lesion scores and higher viral copy numbers compared to line N. Interestingly, line N showed greater down-regulation of B cell related genes (BLNK, TNFSF13B and CD72) compared to line P. While up-regulation of T-cell related genes (CD86 and CTLA4) and Th1 associated cytokines (IFNG, IL2, IL12A and IL15) were documented in both lines, the expression levels of these genes were different in the two lines. Meanwhile, the expression of IFN-related genes IFNB, STAT1, and IRF10, but not IRF5, were up-regulated in both lines. The expression of pro-inflammatory cytokines (IL1B, IL6, IL18, and IL17) and chemokines (CXCLi2, CCL4, CCL5 and CCR5) were up-regulated in both lines with greater increase documented in line P compared to line N. Strikingly, the expression of IL12B was detected only in line P whilst the expression of IL15RA was detected only in line N. In conclusion, the bursal immunopathology of IBDV correlates more with expression of proinflammatory response related genes and does not related to expression of B-cell related genes.
\end{abstract}

Keyword: IBDV; Very virulent; Inbred lines; Immune-related genes 